\title{
Hearing Loss in Children
}

\author{
Andrea L.O. Hebb, MSc, PhD, RN*, Laura MacKenzie and Hannah Wade
}

Department of Psychology, Saint Mary's University, Nova Scotia, Canada

Received: 制October 13, 2018; Published: 㘹 October 17, 2018

*Corresponding author: Andrea L.O. Hebb, MSc, PhD, RN, Department of Psychology, Saint Mary's University, Nova Scotia, Canada

\section{Opinion}

In Canada, more than 2000 children are born with some form of hearing loss (HL) each year, making HL one of the most common birth defects for which screening is available [1]. Categories of hearing loss range from disability in one or two ears, to the type of hearing loss (sensorineural, permanent conductive, neural hearing disorders and mixed), and then to the level of hearing loss [2]. Most research in hearing impairment is focused on the effects and causes of hearing loss, instead of the classification. In Canada, five provinces, Prince Edward Island, New Brunswick, Nova Scotia, Ontario and British Columbia, require universal hearing screening when a baby is born [3].

HL can be classified as one of three basic types: conductive hearing loss (CHL), sensorineural hearing loss (SNHL), and mixed hearing loss [4]. CHL originates in the outer and middle ear and occurs when there is interference with the mechanical transmission of sound, such as middle ear inflammatory infections. SNHL originates in the inner ear and occurs when there is a failure to transduce vibrations to neural impulses in the cochlea. Mixed HL occurs when both CHL and SNHL are present. Auditory processing disorders can be considered a fourth type of HL and occur when there are defects in the brain stem or higher processing centers of the brain [5]. The severity of HL varies from child to child, but the degree of HL can be labeled as slight, mild, moderate, moderately severe, severe, or profound [2]. HL can also be classified as unilateral, such that the impairment occurs in one ear, or bilateral, such that the impairment occurs in both ears [6]. By correctly classifying the type of HL present in a child, caregivers, professionals, and many others can work together to ensure the child receives proper treatment and services to improve quality of life.

\section{Effects of Hearing Loss on Development}

It is important during the diagnosis of hearing loss to classify the type and level of hearing loss the child has, as intervention methods are different based on the degree of hearing loss diagnosed $[3,7]$. Both CHL and SNHL are associated with language and behavioral problems, making early detection and classification critical to the child's cognitive, speech, and language development
$[5,8]$. Deficits in hearing and language development may prove to be a disadvantage to the child in an academic setting if they are not given the proper attention and care [2]. Besides hearing ability, a child with SNHL, the most common type of HL, can be at risk of developing motor, balance, and postural deficits which are common consequences associated with SNHL and its related vestibular abnormalities [4].

\section{Causes of Hearing Loss in Children}

CHL and SNHL can be caused by a variety of congenital factors, such as perinatal hypoxia and prematurity, or acquired factors, such as middle ear infections or bacterial meningitis [5]. Further, causes can be classified as prenatal, which includes maternal drug and alcohol use and in utero infections, perinatal, which includes ototoxic medication and craniofacial abnormalities, and postnatal, which includes trauma to the head and noise exposure [5].

In some cases, it is difficult to determine whether a child's HL is congenital or acquired, thus it is important to take into consideration personal and family history when determining the etiology of HL Ječmenica and colleagues [6] found that autosomal recessive HL was found in 56\% of patients whereas Paludetti and colleagues [5] put forth that autosomal recessive transmission occurs in approximately $80 \%$ of HL cases. Mutations in connexin 26, a gene responsible for encoding a transmembrane protein in gap junctions and $\mathrm{K}+$ channels in the auditory cell receptors, typically give rise to autosomal recessive HL. The mutations can cause high levels of toxic $\mathrm{K}+$ in the hair cells, ultimately leading to irreversible sensory receptor damage [6].

HL is associated with several syndromes, such as Usher syndrome, an autosomal recessive disorder that is the most common cause of deafness associated with blindness [6]. Many common screening tests, such as chorionic villus sampling, amniocentesis, or maternal serum screening can help to detect these syndromes as well as the associated HL. Non-syndromic SNHL can also be detected prenatally using chorionic villus sampling and amniotic fluid sampling. In one study, 30 of the 54 families that had children with non-syndromic SNHL were found to carry gene mutations 
associated with autosomal recessive HL [9]. It is reasonable to assume that prenatal detection of HL would prompt caregivers of children to seek information and resources to promote the overall health and well-being of the developing child.

\section{Treatment of Hearing Loss}

There are several forms of treatment for HL. Hearing aids, a type of amplification device, can be beneficial to children with moderate to severe forms of HL. When an infant or young child is diagnosed with HL, a behind-the-ear hearing aid is often suggested [5]. Cochlear implants are a common treatment for HL and are one of the most effective ways used to correct severe acoustic damage $[4,5]$. Cochlear implants are composed of two components: an external sound processor, which is used to collect and process environmental sounds, and an implanted part, which receives the environmental stimuli delivers it directly to nerve fibers in the form of electrical signals. The inner component of cochlear implants is designed to bypass the nonfunctioning auditory receptors [5]. Other treatments include bone-anchored hearing devices and auditory brainstem implants, which are similar to cochlear implants and used to treat auditory processing disorders, yet the latter has been associated with significant morbidity and mortality [5].

Today, with the access and improvement of hearing assisted devices, hearing can be enhanced. However, it is important to note that hearing aids and cochlear implants require an adjustment period and consistent use for brain reorganization and adaptation to newly acquired sounds. There are different hearing assisted devices used based on the type of hearing loss. For example, bone anchored hearing aids are used for children that have permanent conductive, hearing loss or unilateral deafness [7]. Cochlear implants are used to restore hearing when the inner ear is damaged or non-functioning, while traditional over the ear hearing aids simply amplify sound [7]. While hearing aids amplify all sounds in the individual's environment, hearing aids can be connected to frequency modulator (FM) systems which are useful for school aged children in the classroom. FM systems direct the teacher's voice to the student's hearing aid reducing or eliminating any potential distracting background noise. Often a measure used by audiologists of the efficacy of hearing assisted technology, is school performance as well as language proficiency [7].

\section{Benefit of Early Diagnosis}

The benefit of early diagnosis is implementation of intervention programs in a timelier manner to promote integration into the public school system [7]. Early intervention by 6 months of age was associated with a positive effect on acquisition and use of expressive language in later childhood while delayed intervention after 1 year of age resulted in language delay $[3,10]$. Rehabilitation services vary depending on the child and degree of HL, but most cases focus on developing language (including sign language), training in listening, and developing proper methods for using hearing aids and assistive devices [5]. Vestibular rehabilitation and physical therapy have a positive effect on balance outcomes for children suffering from SNHL related vestibular issues [4].

Although treatment is crucial for a child diagnosed with HL, proper services must be available to the family to support the child. In Nova Scotia, the Society of Deaf and Hard of Hearing is committed to providing services to meet the needs of those affected by HL. Services include providing sign language interpreters, self-help support groups, and public workshops [11]. The Deaf and Hard of Hearing Youth Association of Nova Scotia aims to help youth reach their full potential by helping to develop language, social skills, and healthy relationships. The association allows youth to meet with other youth living with HL and hopes to promote youth with hearing impairments to become leaders and positive role models for younger children with HL [12].

Through early detection and classification of HL implementation of appropriate intervention programs such as speech and language therapy and hearing assisted technology can facilitate language and communication to minimize negative long-term sequelae on development.

\section{References}

1. Hearing Foundation of Canada (2018) Newborn Hearing Screening. (n.d.). hearingfoundation.ca.

2. Clark JG (1981) Uses and abuses of hearing loss classification. American Speech Hearing Association 23(7): 493-500.

3. Eskander, Papsin (2014) Screening infants for hearing impairment in Canada. Canadian Medical Association Journal 186(14): 1048-1049.

4. Fernandes R, Hari prasad S, Kumar VK (2015) Physical therapy management for balance deficits in children with hearing impairments: A systematic review. Journal of Paediatrics and Child Health 51(8): 753758.

5. Paludetti G, Conti G, Di Nardo W, De Corso E, Rolesi R, et al. (2012) Infant hearing loss: From diagnosis to therapy Official Report of XXI Conference of Italian Society of Pediatric Otorhinolaryngology. Acta Otorhinolaryngologica Italica, 32(6): 347-370.

6. Ječmenica J, Bajec-Opančina A, Ječmenica D (2015) Genetic hearing impairment. Child's Nervous System 31(4): 515-519.

7. Cole E, Flexer, Carol Ann (2011) Children with hearing loss: Developing listening and talking, birth to six ( $\left.2^{\text {nd }} e d n\right)$. Plural Pub, San Diego, Canada.

8. Martines F, Martines E, Mucia M, Sciacca V, Salvago P (2013) Prelingual sensorineural hearing loss and infants at risk: Western Sicily report. International Journal of Pediatric Otorhinolaryngology 77(4): 513-518.

9. Yin A, Liu C, Zhang Y, Wu J, Mai M, et al. (2014) Genetic counseling and prenatal diagnosis for hereditary hearing loss in high-risk families. International Journal of Pediatric Otorhinolaryngology 78(8): 13561359.

10. Kitsko D (2014) Pediatric sensorineural hearing loss. Ear, Nose \& Throat Journal 93(6): 196198.

11. About Us. (2015) Canadian Association of the Deaf.

12. About Us. (n.d.). Society of Deaf and Hard of Hearing Nova Scotians. 
(C) (P) This work is licensed under Creative

To Submit Your Article Click Here: $\quad$ Submit Article

DOI: 10.32474/LOJNHC.2018.02.000126

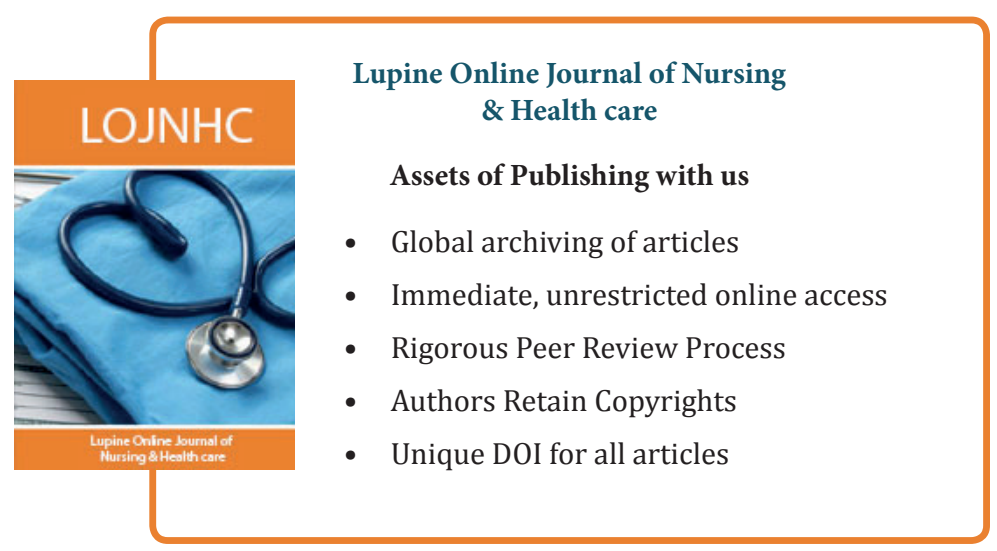

